\title{
Avaliação de empoderamento: considerações teórico-metodológicas aplicadas ao campo da saúde
}

Empowerment evaluation: theoretical and methodological considerations applied to the health area Evaluación de empoderamiento: consideraciones teóricas y metodológicas aplicadas al campo de la salud

Elitiele Ortiz dos Santos ${ }^{1}$, Leandro Barbosa de Pinhoo ${ }^{1}$, Adriane Domingues Eslabão ${ }^{1}$, Rafael Gil Medeiros ${ }^{1}$, Talita Portella Cassola ${ }^{1}$

Como citar este artigo:

Santos EO, Pinho LB, Eslabão AD, Medeiros RG, Cassola TP. Empowerment evaluation: theoretical and methodological considerations applied to the health area. Rev Esc Enferm USP. 2018;52:e03400. DOI: http://dx.doi.org/10.1590/S1980-220X2017035403400

${ }^{1}$ Universidade Federal do Rio Grande do Sul, Escola de Enfermagem, Porto Alegre, RS, Brasil.

\begin{abstract}
The objective of this article is to discuss the empowerment evaluation as a theoretical and methodological framework and its applications to the health area. This is a theoretical article that presents the conceptual and methodological aspects and the principles of the empowerment evaluation. This evaluation is regarded as an approach that allows the participation of interest groups in the process, in order to improve the qualification and transformation of the knowledge and practices of the context evaluated. In the health area, the possible articulations between this framework and the complexity of the practices are discussed, aiming to increase the sense of belonging and responsibility of the participants in the evaluation process. Empowerment evaluation is a viable and powerful theoretical and methodological alternative in the health area, which, in addition to producing knowledge applied to the reality of interest groups, also provides a better understanding of work processes and institutional arrangements in the field of health management and health care.
\end{abstract}

DESCRIPTORS

Health Evaluation; Process Assessment; Health Management; Health Policy. 


\section{INTRODUÇÃO}

A avaliação de empoderamento é uma proposta recente disparada em 1993 no encontro da Associação Americana de Avaliação (American Evaluation Association) e representa um avanço em relação aos referenciais teórico-metodológicos de avaliações tradicionais. Trata-se de uma avaliação participativa e organizada de forma estratégica para que os diferentes grupos de interesse (stakeholders) se apropriem do processo avaliativo e desenvolvam uma intervenção no próprio serviço ou programa em avaliação ${ }^{(1)}$.

No Brasil, o campo da avaliação em saúde teve maior visibilidade a partir de 1980, junto às experiências do Movimento da Reforma Sanitária, no cenário de um país em processo de democratização e reformulação do sistema de saúde. Esse movimento, ao mesmo tempo, que ampliou a importância econômica e política dos serviços e programas, trouxe também questionamentos sobre a eficiência, eficácia e efetividade das ações, incitando a necessidade de avaliações sistemáticas na área da saúde. Trata-se de um campo marcado de tensões e interesses políticos, sintonizado com a tendência internacional que inclui a avaliação como importante componente da gestão sanitária e fundamental instrumento para a tomada de decisão em relação à reorganização dos investimentos públicos ${ }^{(2-3)}$.

As práticas avaliativas na área da saúde, muitas vezes vinculadas às pesquisas científicas, permitiram uma melhor compreensão do funcionamento dos serviços e programas e da forma de desenvolvimento do cuidado por diferentes profissionais, resultando em um importante panorama da forma como os serviços operam no Sistema Único de Saúde (SUS), seus desafios de estrutura, processo de trabalho e resultados das ações ${ }^{(4)}$. A participação de diferentes grupos de interesse no processo avaliativo (usuários, familiares, trabalhadores e gestores), que com suas diferentes percepções e valores ampliam o eixo pelo qual os julgamentos são emitidos, trouxe novas perspectivas para o campo da avaliação. Nesse sentido, outros questionamentos e desafios que problematizam a função das práticas avaliativas na saúde foram capazes de impulsionar mudanças ou transformações.

Do ponto de vista teórico, a produção em torno da avaliação nos últimos anos pode ser dividida em três direções: avaliações voltadas para o método, para o valor e para o uso. $\mathrm{Na}$ direção do método, encontram-se aquelas avaliações especialmente ocupadas com o rigor do método, com vistas à generalização do processo avaliativo. Já as avaliações voltadas para o valor incluem iniciativas de avaliação que enfatizam os valores e julgamentos. São avaliações que visam a contemplar as necessidades e perspectivas de diferentes grupos de interesse envolvidos com determinado serviço ou programa avaliado ${ }^{(5)}$.

As avaliações direcionadas ao uso, por sua vez, são aquelas especialmente preocupadas com a efetiva utilização dos resultados da avaliação. Nesses estudos, os resultados das avaliações buscam subsidiar a tomada de decisão, por meio da valorização das necessidades e questões dos grupos de interesse. Esses estudos utilizam métodos orientados para o uso dos resultados da avaliação, principalmente pelas pessoas diretamente envolvidas com o objeto avaliado ${ }^{(5)}$.
As avaliações voltadas para o valor, entre elas a abordagem de quarta geração, foi um marco paradigmático inovador no campo da avaliação em saúde por introduzir o desenvolvimento de processos mais democráticos e participativos com a incorporação do julgamento de valor de diferentes grupos de interesse a partir de uma perspectiva construtivista $^{(6-9)}$. Nesse sentido, constituiu-se em um novo desafio para o campo da avaliação, que, até então, era muito voltado para pesquisas avaliativas centradas na mensuração.

Entretanto, atualmente, há uma necessidade candente de avaliações direcionadas para a utilização dos resultados do processo avaliativo, ou seja, avaliações que coloquem em análise o processo que possibilita fazer tal julgamento, $o$ envolvimento dos atores e o uso da informação de forma coletiva, para compreender o objeto em avaliação e impulsionar propostas de mudanças que possam qualificá-lo. Nesse sentido, nasce a riqueza dos processos interativos e formativos da avaliação, visando à qualificação das práticas, de programas e a construção do conhecimento no campo das pesquisas sociais. Não descartaremos a importância das avaliações tradicionais, mas no campo do conhecimento urge a necessidade de revisitar processos para aprimorar as práticas.

Diante disso, apresentaremos, nesse estudo, os caminhos de uma nova abordagem de avaliação, conhecida como avaliação de empoderamento (Empowerment Evaluation), uma proposta direcionada para o uso do processo avaliativo que vem redimensionando a discussão em torno das políticas e práticas participativas no campo. Entendendo que essa avaliação tem por objetivo promover a apropriação das etapas da avaliação pelos diferentes grupos de interesse, a utilização dos dados do processo para a tomada de decisão e a ação na realidade para a transformação e qualificação das práticas, destacaremos neste artigo sua relevância para o campo da saúde.

Portanto, o objetivo deste estudo é discutir a avaliação de empoderamento, atrelando-a à proposta de qualificação do cuidado na área da saúde, e não somente ao julgamento de valor do objeto avaliado, ou a um processo descritivo dos serviços, dos programas e das práticas, conforme vem sendo marcado o campo da avaliação em saúde. Propomos, neste estudo, repensar a avaliação, redirecionando o olhar para o uso do processo avaliativo na sua função formativa, enquanto dispositivo que possibilita reflexão, crítica e tomada de decisão, impulsionando transformações nos processos de trabalho para a qualificação das práticas de cuidado em saúde.

\section{AvaliaÇão de Empoderamento}

A avaliação de empoderamento se apresenta como um referencial teórico e metodológico para avaliar programas e serviços em diferentes áreas, definida como o uso de conceitos, princípios, técnicas e resultados para promover aprimoramento (improvement) e autodeterminação (self-determination $)^{(1)}$ dos grupos de interesse.

O conceito de empoderamento é complexo e apresenta noções de distintas áreas de conhecimento, tendo suas raízes nas lutas pelos direitos civis ${ }^{(10-11)}$. Atualmente, difundiu-se duas noções distintas sobre o conceito empoderamento, a dimensão psicológica e a comunitária. Na dimensão psicológica, encontra-se uma perspectiva filosófica individualista, de 
maior controle do indivíduo sobre a própria vida, que "tende a ignorar a influência dos fatores sociais e estruturais, uma visão que fragmenta a condição humana no momento em que desconecta o comportamento dos homens do contexto sociopolítico em que eles se encontram inseridos"(11).

A concepção de empoderamento adotada neste estudo é a do empoderamento comunitário, compreendido como um processo que enseja a construção de estratégias que promovam a participação dos indivíduos e coletivos na análise e atuação nos problemas do seu meio, considerando criticamente as questões sociais e políticas envolvidas. Tais estratégias visam a apoiar a organização de uma estrutura propícia para os processos de reflexão e diálogo sobre as questões postas na sociedade, podendo trazer subsídios para a conscientização das situações, capacidade de decisão e intervenção de forma participativa e crítica ${ }^{(11)}$.

Assim, empoderamento, termo traduzido diretamente do inglês, empowerment, é definido como um processo "pelo qual as pessoas ou comunidades adquirem maior controle sobre as decisões e ações que afetam sua saúde" ${ }^{(12)}$. A partir dessa perspectiva, destaca-se a importância estratégica do empoderamento no campo da avaliação em saúde. Nessa linha, a avaliação de empoderamento é compreendida na sua dimensão de autodeterminação, relacionada com a capacidade de os indivíduos e comunidades exercerem, por meio da avaliação, influência sobre as decisões que os afetam, identificando e expressando suas necessidades, seus recursos, estabelecendo objetivos, planos de ação, avaliação de resultados e, consequentemente, mudanças e melhorias em projetos, programas e outras tecnologias sociais ${ }^{(1)}$.

$A$ avaliação de empoderamento envolve julgamento e ação, assim, é preciso conhecer, interagir, analisar e agir. Há quatro dimensões que caracterizam o processo avaliativo $\left.{ }^{(13)}: 1\right)$ dimensão propositiva - a pesquisa e a avaliação são para a ação e não somente para a compreensão dos fatos e da realidade; 2) dimensão epistemológica - a ação produz conhecimento, ou seja, gera aprendizado ao exercê-la; 3 ) dimensão contextual - há uma necessidade de compreender que os contextos locais são únicos e que generalizações devem ser emitidas com cautela; 4) dimensão relacional - avaliador e grupos de interesse são agentes do processo, portanto, não são neutros ${ }^{(13)}$.

O processo participativo não se restringe à observação do pesquisador ou ao fornecimento de informações a respeito do campo da pesquisa, mas abrange também um envolvimento efetivo de participantes implicados em uma ação. $\mathrm{O}$ avaliador, também chamado de critical friend, atua como um facilitador, que apoia sem dominar o diálogo e as condições do processo, colaborando com informações e conhecimentos, garantindo o direito de fala e manifestação dos grupos de interesse ${ }^{(1)}$.

A Avaliação de Empoderamento é orientada por 10 princípios, que devem estar presentes na construção teórica e na prática do processo avaliativo. Esses princípios são qualificados como "lentes" para focar a avaliação(1). São eles: 1. Aprimoramento (Improvement): Refere-se à utilização de ferramentas e métodos para auxiliar as pessoas, programas e organizações a aprimorar o objeto avaliado.

2. Apropriação por parte da comunidade (Community ownership): Valoriza e facilita a apropriação pelos stakeholders do processo avaliativo.
3. Inclusão (Inclusion): Convite ao envolvimento e participação dos diferentes grupos de interesse envolvidos com o objeto avaliado.

4. Participação democrática (Democratic participation): Busca garantir o direito à fala e opinião dos diferentes stakeholders. Este princípio está articulado à transparência, clareza e confiança.

5. Justiça social (Social justice): Envolve o reconhecimento das desigualdades sociais e a necessidade de melhorar essas condições. A ideia de justiça social também se refere ao compromisso com a seleção e uso adequado de ferramentas e métodos flexíveis ao contexto. Além disso, é um princípio que precisa incorporar contribuições sociais, adaptado a cada realidade.

6. Conhecimento da comunidade (Community knowledge): Reconhece e valoriza o conhecimento dos stakeholders como útil e válido. Além disso, estimula o uso do conhecimento e dos saberes.

7. Estratégias baseadas em evidências (Evidence-based strategies): Refere-se à valorização de estratégias baseadas em evidências científicas. $\mathrm{O}$ conhecimento científico e o conhecimento da comunidade devem ser valorizados como fundamentais para compor o processo avaliativo e viabilizar o compartilhamento, para novas sínteses do conhecimento.

8. Desenvolvimento de capacidades (Capacity building): Designação de instrumentos ou meios para apoiar os stakebolders a melhorar o planejamento e execução do objeto avaliado.

9. Aprendizagem organizacional (Organizational learning): Refere-se ao aprendizado possibilitado pela utilização de ferramentas e métodos no processo avaliativo. Envolve a experiência de participação no processo, com base nos erros, sucessos e correções no meio do caminho.

10. Responsabilização (Accountability): Envolve a prestação de contas à sociedade e aos stakeholders. A geração de dados do processo e a orientação para a utilização dos resultados é uma via para a prestação de contas ao público. A configuração dos princípios contribui para a qualidade da avaliação. Entretanto, nem todos os princípios estarão presentes com a mesma relevância, pois são condicionados ao contexto local e ao propósito da avaliação.

A avaliação de empoderamento idealmente propõe a institucionalização da avaliação, ou seja, que a avaliação não se realize de forma esporádica, mas que seja sustentada como parte de uma estratégia de gestão, baseada na autodeterminação e na capacidade de construção dos atores sociais. $\mathrm{O}$ desenvolvimento da avaliação de empoderamento prevê um contínuo processo, com ciclos interativos de reflexão e ação e com ajustes sempre que necessários. Acredita-se que o mérito e o valor não são verdades estáticas, pois as populações, os objetivos e os conhecimentos sobre práticas de programas mudam. Diante disso, sugerem-se avaliações contínuas, com a redefinição de metas e planos de ação( ${ }^{(1)}$.

Com relação à operacionalização, a avaliação de empoderamento é desenvolvida em um fórum aberto e participativo, com a presença dos grupos de interesse envolvidos com o objeto avaliado, convidados a apresentar e discutir suas 
concepções. O método abrange algumas etapas: em primeiro lugar, deve-se compreender a posição atual do objeto avaliado, seus pontos fortes e fracos; a etapa seguinte consiste em estabelecer metas, determinando para onde o programa/serviço deseja ir e enfatizando explicitamente sua melhoria. Em seguida, é preciso definir estratégias para atingir os objetivos e metas do programa/serviço. Por fim, é preciso determinar quais tipos de evidências são necessárias para documentar o progresso para atingir objetivos. $\mathrm{Na}$ sua formulação mais recente, as etapas supracitadas foram combinadas em três ${ }^{(1)}$ : a. A primeira consiste em estabelecer a missão (mission) da avaliação. São considerados os valores, as percepções, as opiniões de cada participante com relação ao que entendem ser importante para o propósito da missão. Constrói-se um texto único, de consenso, que sintetiza a missão da avaliação, ou seja, o que é preciso qualificar e melhorar no serviço ou programa?

b. A segunda etapa consiste em tomar conhecimento (take stock) da situação atual do objeto avaliado, identificando e priorizando suas atividades mais significativas para o alcance da missão. A partir desse panorama, os participantes são estimulados a selecionar as atividades prioritárias, que não necessariamente são aquelas atividades que enfrentam mais dificuldades, mas aquelas que entendem ser mais necessárias e, sobretudo, que estejam dispostos a investir nelas, desenvolvendo ações para melhorá-las.

c. Por fim, estabelece-se um curso de planejamento para o futuro (planning the future) definindo objetivos e estratégias para atingir as atividades elencadas como prioridades, cuidando-se também para estabelecer as evidências necessárias para monitorar os progressos. Os objetivos retratam o que se pretende alcançar a partir do patamar que se encontra determinada atividade e, deste modo, aprimorar tanto o que já se faz quanto o que necessita de melhorias. $\mathrm{Na}$ definição dos objetivos, devem ser levadas em conta as distintas perspectivas colocadas em discussão, sem deixar de ter o caráter realista, considerando sempre as condições iniciais, os recursos, o tempo, a motivação, os talentos e a dinâmica das relações em campo. O diálogo é uma das partes mais importantes da avaliação. As motivações que levaram a determinadas opiniões e a utilização de evidências são usadas para dar suporte e promover mais foco nas atividades de planejamento para o futuro.

Essa avaliação visa a desenvolver um processo participativo, colaborativo e democrático, propício para o empoderamento, no qual as pessoas envolvidas são convidadas a exercer o poder para a tomada de decisão no processo avaliativo e na qualificação de questões prioritárias. É uma abordagem que garante possibilidades de decisão sobre os aspectos relacionados à avaliação, além de oferecer aportes para a construção de uma ação coletiva significativa e relevante. Assim, cria caminhos para o debate, no qual as transformações sobre as práticas partem da análise coletiva de questões cotidianas e necessidades dos atores.

\section{INTERFACES DA AVALIAÇÃO DE EMPODERAMENTO COM O} CAMPO DAS POLÍTICAS E DAS PESQUISAS AVALIATIVAS EM SAÚDE

A política nacional de saúde, por sua concepção e desenvolvimento, é participativa, abrindo possibilidades para que os agentes façam discussões sobre os processos de gestão das políticas públicas. A participação e o empoderamento das pessoas na gestão, no planejamento e na avaliação dos programas de saúde devem ser vistos como componentes vitais para a sustentação da própria política pública. Desse modo, a avaliação de empoderamento vem colaborar com processos de gestão e de assistência que se pretendem participativos e democráticos, capazes de sustentar e fortalecer as ações e programas de saúde no atual contexto político.

A Avaliação de Empoderamento já vem sendo operacionalizada no contexto nacional e internacional em estudos voltados para as áreas da saúde com diferentes públicos, incluindo pessoas com doenças infectocontagiosas ${ }^{(14)} \mathrm{e} \mathrm{ex-}$ -detentas ${ }^{(15)}$, e em diferentes esferas, como na prevenção e assistência à violência sexual ${ }^{(15-17)}$, educação nutricional ${ }^{(18)} \mathrm{e}$ uso de estratégias psicoeducativas ${ }^{(19)}$.

Um estudo realizado em Quebec utilizou a avaliação de empoderamento para qualificar as práticas de saúde ofertadas a pessoas com Hepatite C. Primeiramente, identificou-se que o acesso e o tratamento da Hepatite $\mathrm{C}$ não eram efetivos e enfrentavam problemas de ordem organizacional das equipes. A abordagem avaliativa envolvou fases de discussão com os trabalhadores de uma organização de serviços de Hepatite C. Foram realizadas diferentes atividades coletivas de construção do conhecimento, na qual se identificou as necessidades das pessoas com Hepatite $\mathrm{C}$, os recursos disponíveis, as facilidades, os limites e as experiências ao longo de um percurso de prevenção, triagem e tratamento ${ }^{(14)}$.

$\mathrm{O}$ uso da abordagem possibilitou reunir as impressões, interpretações e sugestões dos grupos de interesse e, ao mesmo tempo, o aprendizado das equipes. Nas sessões de discussão, foram identificadas soluções viáveis para melhorar o acesso à saúde das pessoas com Hepatite $\mathrm{C}$ e priorizar determinadas ações. Nesse sentido, o estudo demonstrou que a avaliação de empoderamento é organizada de tal forma que se torna em si mesmo intervencionista, pois permitiu que os participantes utilizassem o processo avaliativo para construir e compartilhar o conhecimento a respeito da situação-problema, e, sobretudo, pelo impacto transformador na organização dos serviços, impulsionou as pessoas a agirem na realidade por meio do desenvolvimento de intervenções prioritárias ${ }^{(14)}$.

Com relação aos limites da avaliação, os autores destacam a necessidade de fundamentação teórica e práticas que contribuam para essa agenda de pesquisas avaliativas, além de estudos que se dedique a estratégias metodológicas que possam acompanhar a efetividade das metas propostas ${ }^{(14)}$.

Em outro estudo na área da saúde, a avaliação de empoderamento foi utilizada com o objetivo de contribuir para a institucionalização da avaliação, ou seja, a construção da capacidade de avaliação contínua de um programa de prevenção à violência sexual. A experiência da avaliação possibilitou o aprendizado sobre o processo, com a identificação de aspectos particularmente úteis para alcançar resultados, incluindo o investimento em relações colaborativas, maximização da participação entre os membros do programa, adaptações de conteúdos e técnicas de acordo com as especificidades, combinando estrutura de aprendizado e utilização de ações adaptadas e prioritárias ao contexto ${ }^{(20)}$. 
Os programas participantes relataram melhoria nas suas habilidades de avaliação, passaram a otimizar os recursos dedicados à avaliação, assim como a extensão e sofisticação de sua prática avaliativa, resultando em diferentes melhorias dos programas, tanto na parte de recursos humanos como nas questões burocráticas. Assim, fortaleceu-se uma cultura organizacional, na qual a avaliação se tornou um componente essencial do trabalho ${ }^{(20)}$.

$\mathrm{Na}$ área da saúde mental, os fundamentos da avaliação de empoderamento foram utilizados em uma pesquisa na Bélgica, encomendada pelo governo com a tarefa de apoiar uma política eficaz baseada em evidências. $\mathrm{O}$ estudo foi mais especificamente voltado para a análise de um processo de implementação de ações psiocoeducativas para redução do estresse, prevenção da depressão e ansiedade. O objetivo era determinar se uma intervenção psicoeducativa poderia ser um subsídio para as organizações de atenção primária de saúde mental conseguir reduzir a carga crescente de problemas nessa área ${ }^{(19)}$.

Os resultados do referido estudo problematizaram principalmente o fato de a abordagem participativa levar a uma melhor compreensão da intervenção, dos objetivos e dos elementos centrais pelos agentes de saúde locais que a implementaram, estimulando-os a desenvolver, adaptar e implementar futuras intervenções. $\mathrm{O}$ foco do estudo foi demonstrar como uma intervenção pode se beneficiar de adaptações guiadas pelos conhecimentos locais, mantendo os principais componentes do programa e, ao mesmo tempo, respeitar a fidelidade de implementação. A eficácia da intervenção ocorreu pelas possibilidades de os parceiros locais adaptar uma intervenção às suas necessidades ${ }^{(19)}$.

No contexto brasileiro, identificou-se o uso da avaliação de empoderamento em uma pesquisa que avaliou as contribuições da arte/educação para a promoção da saúde de adolescentes em vulnerabilidade social. Com a priorização das atividades de arte e educação como foco para o alcance da missão, esta, construída pelos adolescentes, representou o conceito de promoção de saúde. A partir disso, foram implementadas metas para a saúde por meio de atividades artísticas. No início do processo avaliativo, o grupo apresentava uma visão restrita quanto às atividades de arte/educação, mas ao término da avaliação os adolescentes ampliaram a crítica do olhar avaliativo, que foi direcionado à qualidade no desenvolvimento das atividades, e não apenas à existência destas ${ }^{(21)}$.

As autoras da pesquisa identificaram algumas limitações para a aplicação da Avaliação de Empoderamento, como: a falta de recursos materiais para o desenvolvimento de algumas atividades entendidas como prioritárias, a resistência da instituição a participar de um processo avaliativo e a redução de participantes na etapa de reavaliação. Essas limitações impossibilitaram o alcance das metas elencadas a partir da missão, mas tornou a avaliação propícia para impulsionar reflexões críticas nos participantes e a autopercepção para o alcance de melhorias locais em curto prazo. Contudo, é preciso continuidade para gerar mudanças individuais e coletivas em médio e longo prazo ${ }^{(21)}$.

Portanto, a partir dos estudos referidos, é possível identificar potencialidades, desafios e fragilidades no uso da avaliação de empoderamento. Entre as potencialidades, identifica-se o envolvimento dos diferentes grupos de interesse no processo avaliativo, uma melhor compreensão da avaliação do objeto avaliado e dos elementos centrais para a transformação e qualificação das práticas, a priorização na tomada de decisão, as ações colaborativas e a implementação de intervenções no contexto de trabalho. Além disso, destacam-se a flexibilidade metodológica, que permite diferentes adaptações coerentes aos contextos e necessidades locais.

Com relação aos desafios, é importante a organização do tempo dedicado ao processo avaliativo, uma vez que muitas equipes enfrentam grandes demandas de trabalho, sendo necessário um adequado planejamento do processo. Há profissionais que apresentam resistência para avaliar suas atividades de forma coletiva, pois comumente as avaliações são realizadas de modo individual nos serviços e também pela concepção histórica da avaliação como um ato de julgamento e crítica unicamente. Nesse sentido, a avaliação de empoderamento requer apoio institucional, o comprometimento de todos os envolvidos e sensibilização das equipes sobre o processo avaliativo e sua perspectiva construtiva de qualificação das práticas e melhorias na organização do processo de trabalho.

No que se referem às fragilidades, os estudos destacam a carência de elementos metodológicos que possam de fato acompanhar se o processo avaliativo foi efetivo ao que se propôs, no caso do alcance das metas traçadas e dos princípios que orientam a avaliação, assim, sugerem-se mais estudos com essa temática. Apesar dessa fragilidade destacada, entende-se que a opção pela avaliação de empoderamento não é só para determinar mudanças e impactos mensuráveis, mas também para registrar os movimentos desenvolvidos com a pesquisa avaliativa e promover construções coletivas realizadas junto aos grupos de interesse.

O incentivo à participação crítica por todas as pessoas envolvidas no planejamento e gestão tenciona a cultura da gestão clássica, organizada sob uma perspectiva vertical. A Avaliação de empoderamento pode permitir a reflexão sobre em que medida essa cultura da avaliação e de tomada de decisões verticais influenciam a dinâmica de processos de trabalho coletivos e estratégias avaliativas participativas, situações essas que, muitas vezes, podem estar na raiz dos próprios desafios enfrentados pelas equipes.

É preciso superar a percepção comum do ato avaliativo como sendo uma prática verticalizada. Essa certamente não é uma cultura favorável à melhoria das relações institucionais e, muitas vezes, promove, mesmo como efeito indesejado, a negligência e o sentimento de não pertencimento ao processo em longo prazo. No caso de um serviço de saúde, a qualificação do cuidado ofertado demanda a participação efetiva dos grupos de interesse (usuários, trabalhadores, gestores e sua rede comunitária), de modo a produzir, caso a caso, tendências inovadoras e saídas negociadas para os conflitos ou embates.

Ao pensar mudanças no campo da avaliação, é importante levar em conta que a cultura da verticalização no fluxo avaliativo e deliberativo na gestão do campo da saúde não é um mero resultado das decisões individuais de atuais gestores, 
mas, sim, de todo um histórico institucional, que também é fruto do atravessamento de forças políticas. A adoção de metodologias como a da Avaliação de Empoderamento permite, portanto, falar no empoderamento para além do lócus individual, visando à autonomia das instituições dentro daquilo que é de sua competência. Indicador esse que fala, certamente, do grau de maturidade dos processos democráticos e da sustentabilidade das políticas públicas de saúde.

Nesse sentido, entendemos que essa abordagem também fortalece discussões em torno da institucionalização da prática avaliativa no processo de trabalho, ou seja, a avaliação torna-se um processo estratégico na construção de efetivas e contínuas mudanças assistenciais que superem o término dos cronogramas formais dos projetos.

No que se refere ao contexto da pesquisa avaliativa, entendemos que a avaliação de empoderamento traz importantes subsídios que tangenciam aspectos metodológicos e políticos da avaliação em saúde, fundamentados principalmente em dois aspectos considerados centrais. O primeiro aspecto é a superação de uma visão positivista da avaliação, que parte do princípio da neutralidade do pesquisador em campo, para uma prática avaliativa na qual se acredita que não é possível avaliar algo de forma neutra e objetiva sem afetar também os fenômenos de estudo e o contexto avaliado. Trata-se de reconhecer que a realidade que se busca avaliar já está sendo alterada com a pesquisa.

Nessa abordagem, o pesquisador também é afetado pelo campo, ao mesmo tempo em que o modifica. Tal perspectiva aumenta o grau de comunicação e corresponsabilização da avaliação, em oposição à postura hierárquica do avaliador especialista que planeja, decide e executa a avaliação de forma isolada a priori ${ }^{(22)}$. Com isso, o desafio é colocar em análise a dinâmica das relações provocada por essa interação, as construções conjuntas, as negociações, o gerenciamento de conflitos, os problemas, o consenso e os enfrentamentos que levam à tomada de decisão na avaliação em saúde.

Essa perspectiva evidencia o quanto a metodologia, as questões de pesquisa e a linguagem usada deve ser pensada cuidadosamente, pois afetam usuários dos serviços e equipes na qual é desenvolvida. Nesse sentido, é preciso alcançar um posicionamento construtivo entre pesquisador e os grupos de interesse, de forma que sejam reconhecidos como membros corresponsáveis e importantes na equipe ${ }^{(23)}$.

O segundo aspecto é o empoderamento dos grupos de interesse, pelo qual a pesquisa avaliativa deve garantir uma maior apropriação do processo, incluindo os participantes nas decisões que os afetam, identificando e expressando as questões de interesse, as necessidades, os recursos e as estratégias de mudanças frente aos limites e às dificuldades da prática ${ }^{(1)}$.

É uma abordagem que busca aumentar o poder dos participantes com o processo de avaliação, estimulando-os ao comprometimento com a melhoria das políticas públicas. Também objetiva beneficiar os grupos sociais, uma vez que lhes possibilita compreender melhor os próprios problemas e desafios, buscando, assim, os meios necessários para modificá-los ${ }^{(24-25)}$.
A avaliação, ao integrar os diferentes stakeholders e promover a aprendizagem sobre o objeto avaliado e sobre a avaliação, torna-se um meio de mudanças operacionais no próprio capital avaliado, podendo ter efeitos na resolução dos próprios problemas do contexto. Com isso, a avaliação trabalha na perspectiva de direcionar o potencial de cada grupo de interesse e, assim, construir os meios e instrumentos necessários para buscar as transformações ${ }^{(24)}$.

Diante disso, destacamos que a avaliação não deve se limitar aos erros e às dificuldades, mas focar o que pode ser construído para guiar e impulsionar as práticas futuras $^{(25)}$. Essa perspectiva pode contribuir para superar a ideia de punição e descrédito da avaliação, já que em muitos momentos da história essa concepção foi utilizada para tornar evidente as ineficiências dos programas, das ações e das políticas.

Esses dois aspectos que discutimos, referentes à necessidade de superar a perspectiva avaliativa positivista e caminhar no sentido do empoderamento dos grupos de interesse, são centrais para propor esse referencial avaliativo como dispositivo de qualificação do cuidado na área da saúde. Observadas essas características, trata-se de um dispositivo que produz encontros mais democráticos entre os diferentes grupos de interesse envolvidos com o cuidado em saúde, bem como dispara reflexão da prática, potencializando um campo de possibilidades para qualificar o cuidado, respeitando as diferentes percepções, crenças e culturas.

O cuidado, nesse sentido, é pautado nos princípios do SUS, da integralidade, equidade, e universalidade do acesso, partindo das necessidades locais para ser construído e qualificado. É um campo complexo atravessado pelas políticas públicas, relações de saber-poder e diferentes tecnologias. Portanto, entre tantos elementos importantes nessa composição do cuidado, entendemos a avaliação como um deles, fundamental por sua capacidade de colocar em análise as especificidades locais de construção das políticas públicas e do cuidado em si.

\section{CONSIDERAÇÕES FINAIS}

A avaliação de empoderamento traz contribuições fundamentais para o campo da saúde, na medida em que promove o envolvimento estratégico das pessoas na construção do processo avaliativo e das possíveis soluções para os problemas de sua prática. As particularidades dessa proposta exigem que a avaliação seja adaptada e ajustada ao contexto local com um adequado planejamento, e, sobretudo, que haja genuíno interesse das pessoas envolvidas, já que a utilização da avaliação decorre dessa coprodução do objeto avaliado.

Compreende-se que a qualificação do cuidado a partir da avaliação do empoderamento necessita do aprimoramento de suas estratégias metodológicas para a participação efetiva dos grupos de interesse no processo avaliativo, aumentando-lhes a sensação de pertencimento e responsabilidade pelo processo. A apropriação, pelos diferentes grupos de interesse, dessa abordagem avaliativa e a relação que se pretende com eles e entre eles são diretamente articuladas aos critérios e métodos que se pretende utilizar. Nessa perspectiva, também é necessário que a produção de conhecimento no campo 
da avaliação de empoderamento ultrapasse os muros acadêmicos e envolva o compromisso social, de modo a servir como catalisadora de mudanças nos serviços e nas práticas assistenciais.

Com este estudo, esperamos contribuir para o desenvolvimento contínuo do campo da avaliação de empoderamento, que necessita de avanços na sua compreensão e aplicabilidade. A principal limitação do estudo está relacionada ao fato de haver poucas pesquisas publicadas sobre a utilização dessa avaliação no contexto brasileiro, sobretudo na área da saúde, limitando, assim, a discussão e o aprofundamento dessa temática. No entanto, acredita-se ser inicialmente necessário apresentar elementos teórico-reflexivos, a fim de que essa abordagem seja compreendida, adaptada e aplicada em diferentes contextos de saúde.

Portanto, recomendamos futuros estudos que apresentem reflexão crítica sobre a utilização deste referencial articulado aos referenciais das ciências humanas, sociais e da saúde, bem como aos das políticas de saúde em suas diferentes áreas, principalmente as de maior vulnerabilidade, como a saúde mental, saúde da criança e saúde do idoso, visando a qualificar o cuidado em áreas prioritárias. Além disso, estudos que apresentem resultados sobre a aplicabilidade deste referencial teórico-metodológico, com contribuições para compreender os principais benefícios, limites e desafios dessa abordagem no campo da saúde.

\section{RESUMO}

O objetivo do artigo é discutir a avaliação de empoderamento como referencial teórico-metodológico e suas aplicações ao campo da saúde. Trata-se de um artigo teórico que se propõe a apresentar as raízes conceituais, metodológicas e princípios da avaliação de empoderamento. Considera-se essa avaliação como uma perspectiva que enseja a participação dos grupos de interesse no processo, de modo a auxiliar na qualificação e transformação dos saberes e das práticas do contexto avaliado. No campo da saúde, discutem-se as articulações possíveis entre o referencial e a complexidade das práticas, visando a aumentar a sensação de pertencimento e responsabilidade dos participantes pelo processo avaliativo. A avaliação de empoderamento é uma alternativa teórica e metodológica viável e potente no campo da saúde, que, além de produzir conhecimento aplicado à realidade dos grupos de interesse, também proporciona maior compreensão sobre os processos de trabalho e os diversos arranjos institucionais no campo da gestão e do cuidado em saúde.

\section{DESCRITORES}

Avaliação em Saúde; Avaliação de Processo; Gestão em Saúde; Política de Saúde.

\section{RESUMEN}

El objetivo del artículo es discutir la evaluación de empoderamiento como marco de referencia teórico metodológico y sus aplicaciones al campo de la salud. Se trata de un artículo teórico que se propone a presentar las raíces conceptuales, metodológicas y principios de la evaluación de empoderamiento. Se considera dicha evaluación como una perspectiva que anhela la participación de los grupos de interés en el proceso, de modo auxiliar en la cualificación y transformación de los saberes y las prácticas del contexto valorado. En el campo de la salud, se discuten las articulaciones posibles entre el marco de referencia y la complejidad de las prácticas, con vistas a incrementar la sensación de pertenencia y responsabilidad de los participantes por el proceso evaluativo. La evaluación de empoderamiento es una alternativa teórica y metodológica factible y potente en el campo sanitario, la que, más allá de producir conocimiento aplicado a la realidad de los grupos de interés, también proporciona mayor comprensión acerca de los procesos laborales y los distintos arreglos institucionales en el campo de la gestión y el cuidado sanitarios.

\section{DESCRIPTORES}

Evaluación en Salud; Evaluación de Proceso; Gestión en Salud; Política en Salud.

\section{REFERÊNCIAS}

1. Fetterman DM, Kaftarian SJ, Wandersman A. Empowerment evaluation. Thousand Oaks: Sage; 2015.

2. Bosi MLM, Mercado-Martinez FJ. Avaliação de políticas, programas e serviços de saúde: modelos emergentes de avaliação e reformas sanitárias na América Latina. In: Campos RO, Furtado, JP, organizadores. Desafios da avaliação de Programas e Serviços em Saúde. Campinas: Ed. Unicamp; 2011. p. 41-62.

3. Furtado JP, Silva LMV. A avaliação de programas e serviços de saúde no Brasil enquanto espaço de saberes e práticas. Cad Saúde Pública. 2014;30(12):2643-55.

4. Tanaka OY, Ribeiro EL. Para onde caminhamos com avaliação no Brasil? In: Akerman M, Furtado JP, organizadores. Práticas de avaliação em saúde no Brasil: diálogos. Porto Alegre: Rede Unida; 2015. p. 357-64.

5. Carden F, Alkin MC. Evaluation roots: an international perspective. J MultiDiscipl Eval. 2012;8(7):102-18.

6. Guba EG, Lincoln YS. Avaliação de quarta geração. São Paulo: Unicamp; 2011.

7. Furtado JP. Um método construtivista para a avaliação em saúde. Ciênc Saúde Coletiva. 2001;6(1):165-81.

8. Bard ND, Antunes B, Roos CM, Olschowsky A, Pinho LB. Stigma and prejudice: the experience of crack users. Rev Latino Am Enferm. 2016;24:e2680.

9. Buriola AP, Kantorski LP, Sales CA, Matsuda LM. Nursing practice at a psychiatric emergency service: evaluation using fourth generation assessment. Texto Contexto Enferm. 2016;25(1):1-9.

10. Martins PC, Cotta RMM, Siqueira-Batista R, Mendes FF, Franceschinni SCC, Priore SE, Dias G. Democracia e empoderamento no contexto da promoção da saúde: possibilidades e desafios apresentados ao Programa de Saúde da Família. Physis. 2009;19(3):679-94.

11. Carvalho SR. Os múltiplos sentidos da categoria "empowerment" no projeto de Promoção à Saúde. Cad Saúde Pública, 2004;20(4):1088-95. 
12. World Health Organization (WHO). Glossary of health promotion terms. Geneva: WHO; 1998.

13. Patton MQ. Developmental evaluation: applying complexity concepts to enhance innovation and use. Thousand Oaks: Sage; 2011.

14. Brousselle A, Petitb G, Giraudc MJ, Rietmannd M, Boisverte K, Foleyf V. Using the evaluation process as a lever for improving health and healthcare accessibility: the case of HCV services organization in Quebec. Eval Program Plann. 2016;55:134-43.

15. Pinto RM, Rahman R, Williams A. Policy advocacy and leadership training for formerly incarcerated women: an empowerment evaluation of ReConnect, a program of the Women in Prison Project, Correctional Association of New York. Eval Program Plann. 2014;47:71-81.

16. Cook-Craig PG, Millspaugh PH, Recktenwald EA, Kelly NC, Hegge LM, Coker AL, et al. From empower to Green Dot: successful strategies and lessons learned in developing comprehensive sexual violence primary prevention programming. Violence Against Women.2014;20(10):1162-78.

17. Goodman RM, Noonan R.K. Empowerment evaluation for violence prevention Public Health Programs. Health Promot Pract. 2009;10(1 Suppl):11S-18S.

18. Fourney A, Gregson J, S, Bellow A. Building Evaluation Capacity in Local Programs for Multisite Nutrition Education Interventions. J Nutr Educ Behav. 2011;43(4):130-6.

19. Daele TV, Audenhove CV, Hermans D, Bergh OMD, Broucke SVD. Empowerment implementation: enhancing fidelity and adaptation in a psycho-educational intervention. Health Promot Int. 2012;29(2):212-21.

20. Gibbs DA, Hawkins SR, Clinton-Sherrod AM, Noonan RK. Empowering programs with evaluation technical assistance: outcomes and lessons learned. Health Promot Pract. 2009; 10(1):38-44.

21. Farre AGMC, Pinheiro PNC, Vieira NFC, Gubert FA, Alves MDS, Monteiro EMLM. Promoção da saúde do adolescente baseada na arte/ educação e centrada na comunidade. Rev Bras Enferm; 2018;71(1):31-9.

22. Passo E, Benevides R, Souza TP, Gonçalves LLB, Tallemberg C, Carvalho HF, et al. A dimensão instituinte da avaliação. In: Campos RO, Furtado JP, Passo E, Benevides R, organizadores. Pesquisa avaliativa em saúde: desenho participativo e efeitos da narratividade. São Paulo: Hucitec; 2008. p. 209-30.

23. Lund GE. Fifth-generation evaluation [Internet]. 2012:1-8. http://www.haslebo-partnere.dk/dyn/files/normal_items/427-file/FifthGeneration\%20Evaluation\%20by\%20Gro\%20Emmertsen\%20Lund.pdf

24. Gaëlle B, Eric M. Une approche pluraliste et participative: coproduire l'évaluatio navec la société civile. Rev Informat Soc. 2003;(110):1-7.

25. Hartz ZMA. Avaliação em saúde. In: Pereira IB, Lima JCF, organizadores. Dicionário da educação profissional em saúde. Rio de Janeiro: EPSJV; 2009. p. 1-6. 